\title{
Uso del condón entre adolescentes mexicanos para la prevención de las infecciones de transmisión sexual
}

Cecilia $G$ ayet, $M$ en $D$, (2) Fátima Juárez, PhD, ,1) Laura A. Pedrosa, M en $C,{ }^{(2)}$ C arlos Magis, M en SP.(2)

\begin{abstract}
Gayet C, Juárez F, Pedrosa LA, Magis C. Uso del condón entre adolescentes mexicanos para la prevención de las infecciones de transmisión sexual. Salud Publica Mex 2003;45 supl 5:S632-S640. El texto completo en inglés de este artículo está disponible en: http://www.insp.mx/salud/index.html
\end{abstract}

\section{Resumen}

Objetivo. Investigar el comportamiento sexual actual y el uso de condón en la primera relación sexual de adolescentes de 12 a 19 años de edad, la variación en distintos contextos y la influencia de factores sobre el uso del condón en esa primera relación. Material y métodos. Se utiliza la Encuesta $N$ acional de Salud 2000, y se consideran adolescentes a jovenes de 12 a 19 años de edad, de ambos sexos ( $n=16285)$. Se presenta en primer lugar un análisis descriptivo básico y luego una regresión logística multivariada para determinar la influencia de cuatro tipos de factores (demográficos, socioeconómicos, culturales y cognitivos) sobre el uso de condón en el debut sexual. Resultados Se reporta mayor actividad sexual y un mayor uso de condón entre los hombres y en áreas urbanas. El perfil del adolescente que usó condón en la primera relación es ser adolescente que inicia la vida sexual a una mayor edad, de residencia urbana, que no habla lengua indígena, altamente escolarizado, o de sexo masculino. Conclusiones. Deben diseñarse nuevas políticas de prevención de infecciones de transmisión sexual para cerrar la brecha entre conocimiento y práctica, y dirigidas a los adolescentes que inician su vida sexual más temprano, a los que hablan lengua indígena, a los que viven en áreas rurales, a los menos escolarizados y a las mujeres. El texto completo en inglés de este artículo también está disponible en: http:/l www.insp.mx/salud/index.html

Palabras clave: ITS; comportamiento sexual; ado lescencia; uso de condón; México.
Gayet C, Juárez F, Pedrosa LA, Magis C.

Condom use among Mexican adolescents to prevent sexually transmitted infections.

Salud Publica Mex 2003;45 suppl 5:S632-S640.

The English version of this paper

is available at: http://www.insp.mx/salud/index.html

\begin{abstract}
A bstract
Objective. To investigate the current sexual behavior and condom use during the first sexual intercourse among adolescents, as well as variations and factors influencing condom use at first sexual intercourse. Material and Methods The data source for this study was Mexico's $\mathrm{N}$ ational Health Survey 2000 . Study subjects were male and female ado lescents aged 12 to 19 years $(n=16,258)$. Statistical analysis consisted of descriptive statistics and multivariate lo gistic regression, to assess the asso ciation of four types of factors (demographic, socioeconomic, cultural, and cognitive) with condom use during the first sexual intercourse. Results. Males and residents of urban areas reported greater sexual activity and condom use. Typically, adolescents who used condoms during the first sexual intercourse were male,older, resided in urban areas, non-speakers of an indigenous language, and with higher schooling. Conclusions $\mathrm{N} \mathrm{ew}$ policies should be framed to prevent sexually transmitted infections to span the gap between knowledge and practice, targeting ado lescents starting sexual activity earlier, those who speak an indigenous language, living in rural areas, with less schooling, and females.The English version of this paper is available too at: http://www.insp.mx/salud/index.html
\end{abstract}

Key words: STI; sexual behavior; adolescence; condom use; Mexico

(1) El Colegio de México. México, DF, México.

(2) Centro $\mathrm{N}$ acional para la Prevención y Control del VIH/SIDA (CEN SIDA). México, DF, México.

Fecha de recibido: 29 de octubre de 2002 - Fecha de aprobado: 11 de septiembre de 2003 Solicitud de sobretiros: Carlos Magis Rodríguez, Centro $\mathrm{N}$ acional para la Prevención y Control delVIH/SIDA, Calzada de Tlalpan 4585 2do. piso, colonia Toriello Guerra, 01450 México, D F, México. Correo electrónico: cgayet@ colmet.mx 
E xisten pocos estudios en México sobre el comportamiento sexual de adolescentes* que consideren en particular la protección de las infecciones de transmisión sexual (ITS). Entre los que existen, como se verá más adelante, las estadísticas resultantes no son convergentes, por lo que se hace necesario avanzar en el análisis de fuentes de datos recientes que permitan obtener una visión general comparable a lo largo del tiempo. La reciente disponibilidad de la Encuesta Nacional de Salud 2000 (ENSA 2000) es una oportunidad para la comprensión del comportamiento sexual de adolescentes mexicanos. Por tales razones, esta investigación busca aportar conocimientos sobre el nivel de actividad sexual de adolescentes mexicanos y el uso de medidas de protección de las ITS, indagando sobre las diferencias en el contexto mexicano.

Existe cada vez mayor evidencia acerca de que lo que suceda en los años de formación de la adolescencia será decisivo durante el resto de la juventud, y tendrá gran influencia en la salud y desarrollo futuro en lo personal, lo económico y lo social. ${ }^{1}$ Entre las medidas que se proponen a adolescentes para prevenir las infecciones de transmisión sexual resalta el uso del preservativo o condón. Diversas investigaciones han mostrado los grados de protección que otorga el condón para cada una de las infecciones de transmisión sexual. ${ }^{2-4}$

De allí surge la necesidad de realizar intervenciones educativas a una edad temprana que permita a los adolescentes comenzar con prácticas protegidas que se conviertan en una conducta constante de prevención en la vida futura. Una base para el diseño de las intervenciones es conocer cuáles son los factores que se asocian con el uso del condón en los adolescentes.

Por otra parte, si bien existe información en general sobre la prevalencia de ITS en distintos grupos de población las estadísticas reflejan lo que acontece con la población joven y adulta, muchas veces con prácticas de riesgo, y no con adolescentes. ${ }^{5-7}$ Parte de la dificultad para conocer la prevalencia de las ITS en adolescentes estriba en que son menores de edad, y existen razones jurídicas que dificultan la obtención de muestras biológicas en este grupo etáreo. Las encuestas suelen presentar cifras a partir del auto-reporte de los adolescentes sobre las infecciones sexuales.

\footnotetext{
* Aún aceptando la dificultad de la definición de la etapa adolescente, hemos considerado para este trabajo el periodo entre los 12 y los 19 años, por las características de la Encuesta Nacional de Salud. Esta Encuesta consideró a la adolescencia de acuerdo al criterio de la OMS entre los 10 y los 19 años, pero sólo realizó las preguntas sobre sexualidad a aquellos que tuvieran más de 12 años.
}

Los resultados de encuestas realizadas con anterioridad no siempre son comparables y, menos aún, coincidentes (cuadro I). En relación con la proporción de adolescentes y jóvenes sexualmente activos, una encuesta realizada en 1985 en la Ciudad de México ${ }^{8}$ informaba que $44 \%$ de los hombres y $13 \%$ de las mujeres de 15 a 19 años de edad eran sexualmente activos. En la Encuesta sobre Sexualidad y Familia en Jóvenes de Educación Media Superior (1988), ${ }^{9}$ el porcentaje de sexualmente activos de 14 y más años de edad es de 38.3\% y 5.4\% para hombres y mujeres, respectivamente. La Encuesta sobre Comportamiento Sexual y Reproductivo de los Adolescentes y Jóvenes del Area Metropolitana de la Ciudad de México (1988) ${ }^{10}$ señala 33.3\% para hombres y $20.3 \%$ para mujeres de edades de 15 a 19 años. Para 1994, la Encuesta sobre Comportamiento Sexual en la Ciudad de México habla de 40.5\% de hombres entre 15 y 19 años de edad sexualmente activos. ${ }^{11}$ La Encuesta para el Programa Gente Joven de MEXFAM ${ }^{12}$ realizada en 1999 , señala $22.3 \%$ de hombres $10.3 \%$ de mujeres de 13 a 19 años de edad sexualmente activos. En el año 2000, el Instituto Mexicano de la Juventud indica que $60 \%$ de los hombres y $51 \%$ de las mujeres del grupo 15-29 años de edad se declararon sexualmente activos. ${ }^{13}$ Una encuesta realizada en el año 2001 en Cuernavaca, que incluyó a jóvenes universitarios de 16 años y más (en la cual $93 \%$ era menor de 26 años de edad), indica que $69.9 \%$ de los hombres y $45.8 \%$ de las mujeres declararon ser sexualmente activos. ${ }^{14}$ Los rangos de edad fluctúan entre las distintas encuestas, lo que produce variaciones en los porcentajes de sexualmente activos. Sin embargo, todas las encuestas muestran que en estas edades es mucho mayor la proporción de hombres sexualmente activos que de mujeres.

En cuanto a la edad promedio de la primera relación sexual, la encuesta realizada por MEXFAM ${ }^{12}$ señala que fue de 15.2 años para los hombres y 15.3 años para las mujeres, ambos para el grupo etáreo de 13 a 19 años. La encuesta sobre los universitarios en Cuernavaca ${ }^{14}$ indica 16.9 años para los hombres y 18.3 años para las mujeres. En una investigación realizada en 1995 sobre adolescentes de 14 a 20 años de edad residentes en áreas marginadas de la ciudad de León, Guanajuato, las edades promedio de inicio encontradas fueron de 16.9 para los hombres y 17.6 para las mujeres. ${ }^{15}$

Para comparar la información de las encuestas sobre uso del condón por parte de adolescentes, además de los diferentes límites de edad, se presenta el problema de las variaciones en la formulación de las preguntas. Algunas encuestas reportan datos sobre uso del condón en todas las relaciones sexuales y otras sobre uso del condón en la primera relación sexual (cuadro I). Considerando la primera relación sexual, en $1985^{8}$ muy 
pocos jóvenes solteros reportaban haber usado condón (sólo $6.8 \%$ y $4.8 \%$ de los hombres y las mujeres, respectivamente); en cambio, la encuesta de MEXFAM ${ }^{12}$ de 1999 indica que dijeron haber usado 43.7\% de los hombres y $19.5 \%$ de las mujeres (de todos los estados civiles); en el contexto de los universitarios de Morelos ${ }^{14}$ las cifras son aún más altas: $50.5 \%$ de los hombres y $42.7 \%$ de las mujeres (de todos los estados civiles). Tomando en cuenta a quienes afirmaron haber usado condón alguna vez, en $1988^{9} 38.8 \%$ de los hombres declaró haberlo usado, y en cuanto al uso en la última relación sexual, en $1994^{11}$ lo hizo $44.4 \%$.

Debido a la divergencia entre los resultados de las encuestas presentadas resultaba necesario contar con nueva información de nivel nacional que reflejara los comportamientos actuales tomando en cuenta la población no considerada de alto riesgo. La ENSA 2000, proporciona información reciente de los adolescentes en México y permite conocer no sólo el nivel nacional sino también las diferencias regionales de algunos de los marcadores de comportamientos sexuales. El objetivo de este artículo es conocer los niveles de actividad sexual y de uso de condón en la primera relación sexual entre los adolescentes del país.

\section{Material y métodos}

El presente trabajo realiza un análisis de la ENSA 2000, realizada por la Secretaría de Salud, en hogares y con representatividad nacional y estatal. El diseño muestral de la ENSA 2000 fue probabilístico, polietápico, estratificado y de conglomerados. ${ }^{16}$ Entre los diferentes módulos con que cuenta la encuesta, escogimos para este trabajo el de adolescentes y el de hogares. El módulo de adolescentes de la ENSA 2000 obtuvo información de hombres y mujeres de 10 a 19 años de edad, pero las preguntas sobre comportamiento sexual y reproductivo fueron aplicadas al grupo de edad 12-19 años, por lo que éste fue el grupo considerado para el análisis que aquí se presenta. El número de casos entrevistados de 12 a 19 años de edad es de 16285 adolescentes, representativos de 17649669 adolescentes, siendo 49\% hombres y 51\% mujeres. Este es el grupo de análisis de esta investigación. Este estudio presenta un análisis exploratorio del inicio de la vida sexual, medido a través de la proporción de adolescentes sexualmente activos, y enfatiza en el grupo de solteros y, para aquellos sexualmente activos, analiza la proporción de uso del condón.

El documento muestra, primero, un análisis descriptivo básico, tanto de inicio de la vida sexual como de protección de las ITS a través del uso del condón para los cuales se utiliza la prueba estadística Pearson de dos colas. En la segunda parte se presenta una regresión logística multivariada para determinar la influencia de cuatro tipos de factores seleccionados (demográficos, socioeconómicos, culturales y cognitivos) sobre el uso del condón en la primera relación sexual. Los datos fueron procesados con el paquete estadístico SPSS, versión 11.0 para Windows.

\section{Resultados}

Los porcentajes del total de adolescentes sexualmente activos muestran que casi no hay diferencia entre hombres y mujeres del grupo de edad 12 a 19 años: 16.8\%

\section{Cuadro I \\ Porcentaje de actividad sexual y uso de condón de la población adolescente y joven, POR SEXO, EN DISTINTAS ENCUESTAS.}

\begin{tabular}{|c|c|c|c|c|c|c|c|c|c|c|c|c|}
\hline & \multicolumn{2}{|c|}{$\begin{array}{c}\text { Ciudad de M éxico, } \\
1985^{\circ}\end{array}$} & \multicolumn{2}{|c|}{$\begin{array}{l}\text { México, } \\
1988^{b}\end{array}$} & \multicolumn{2}{|c|}{$\begin{array}{c}\text { Ciudad de M éxico, } \\
1994^{\circ}\end{array}$} & \multicolumn{2}{|c|}{$\begin{array}{l}\text { México, } \\
\text { 199gd }\end{array}$} & \multicolumn{2}{|c|}{$\begin{array}{l}\text { México, } \\
2000^{e}\end{array}$} & \multicolumn{2}{|c|}{$\begin{array}{l}\text { México, } \\
2000^{e}\end{array}$} \\
\hline & Hombres & Mujeres & Hombres & M ujeres & Hombres & Mujeres & Hombres & Mujeres & Hombres & Mujeres & Hombres & Mujeres \\
\hline & $15-24$ & $15-24$ & $14-19$ & $14-19$ & $15-19$ & $15-19$ & $13-19$ & $13-19$ & $12-14$ & $12-14$ & $15-19$ & $15-19$ \\
\hline Porcentaje de sexualmente activos & 44.0 & 13.0 & 38.3 & 5.4 & 40.5 & - & 22.3 & 10.3 & 1.3 & 1.5 & 42.0 & 26.3 \\
\hline Uso del condón & \multicolumn{2}{|c|}{$\begin{array}{l}\text { En la primera } \\
\text { relación sexual }\end{array}$} & \multicolumn{2}{|c|}{$\begin{array}{l}\text { En alguna } \\
\text { relación sexual }\end{array}$} & \multicolumn{2}{|c|}{$\begin{array}{l}\text { En la última } \\
\text { relación sexual }\end{array}$} & \multicolumn{2}{|c|}{$\begin{array}{l}\text { En la primera } \\
\text { relación sexual }\end{array}$} & \multicolumn{2}{|c|}{$\begin{array}{l}\text { En la primera } \\
\text { relación sexual }\end{array}$} & \multicolumn{2}{|c|}{$\begin{array}{l}\text { En la primera } \\
\text { relación sexual }\end{array}$} \\
\hline Porcentaje que usó condón (total) & & & 38.8 & 23.6 & 44.4 & - & 43.7 & 19.5 & 24.1 & 9.9 & 47.1 & 15.2 \\
\hline Porcentaje que usó condón (solteros) & 6.8 & 4.8 & & & & - & & & 24.8 & 9.8 & 51.9 & 23.9 \\
\hline
\end{tabular}

\footnotetext{
a Fuente: Morris, Leo. "D etermining Male Fertility Through Surveys:Young Adult Reproductive Health Surveys in Latin American" (EncuestaYARHS-CDC 1985)

${ }^{b}$ Fuente: Encuesta N acional sobre Sexualidad y Familia en Jóvenes de Educación Media Superior, Conapo, 19889

' Fuente: Encuesta sobre Comportamiento Sexual en la Ciudad de México, CO N ASIDA, 1994. Cálculos de Fátima Juárez ${ }^{11}$

d Fuente: Encuesta para el Programa Gente Joven de Mexfam, 199912

e.Fuente: Encuesta N acional de Salud 2000, SSA (estimaciones propias)
} 
de los hombres ( $n=7 \quad 769)$ y $15.7 \%$ de las mujeres $(n=8251)$ declararon ser sexualmente activos $(p<0.00)$. Los porcentajes son más bajos si consideramos sólo a los solteros. La proporción de solteros que declaró ser sexualmente activos fue de $14.4 \%$ para hombres $(n=7433)$ y $6.9 \%$ para mujeres $(n=7389)(p>0.00)$.

La actividad sexual presenta una tendencia ascendente con la edad: desde casi sin actividad a los 12 años, a $54 \%$ de los hombres de 19 años y $46 \%$ de las mujeres de esa edad $(p<0.00)$. La edad promedio de la primera relación de los sexualmente activos fue muy similar para hombres y mujeres, tanto de manera general como si consideramos sólo a los solteros. La edad se estimó en 15.7 años para los hombres y para las mujeres, considerando todos los estados civiles, y en 15.6 y 15.4, respectivamente, si consideramos sólo a los solteros.

Asimismo, se observa una gran diferencia en el comportamiento sexual entre ámbitos rural y urbano.
Entre los hombres, son activos en áreas urbanas 19.8\%, $\mathrm{y}$ en áreas rurales $12.6 \%(p<0.00)$. De manera similar, entre las mujeres, son activas $17.6 \%$ en áreas urbanas contra $13.4 \%$ en las rurales $(p<0.00)$. Para profundizar sobre las diferencias en los comportamientos a lo largo del país se agruparon las entidades federativas en ocho regiones, de acuerdo con la propuesta de Bassols Batalla, que fue desarrollada por S Rivera (cuadro II). ${ }^{17}$ Aunque se verifican diferencias entre las regiones en la proporción de sexualmente activos al considerar a la población en general, sin distinguir el estado civil $(p<0.00)$, resulta más relevante observar el nivel de actividad sexual de los solteros, porque la gran mayoría de los casados y unidos son sexualmente activos. Como se observa en el cuadro II, tres regiones destacan por una mayor actividad sexual de los adolescentes solteros, tanto para hombres $(p<0.00)$ como para mujeres $(p<0.07)$ : Región I Noroeste, Región III Noreste y Región V Centro E. Las

\section{Cuadro II}

\section{Porcentaje de adolescentes de 12 a 19 años de edad seXualmente activos solteros} POR GRANDES REGIONES DEL PAÍS

\begin{tabular}{|c|c|c|c|c|c|c|c|c|}
\hline \multirow{6}{*}{ Región } & \multicolumn{4}{|c|}{ Hombres solteros } & \multicolumn{4}{|c|}{ M ujeres solteras } \\
\hline & & & \multirow{2}{*}{\multicolumn{2}{|c|}{$\begin{array}{c}\text { Uso de condón } \\
\text { en la primera relación* }\end{array}$}} & & & \multicolumn{2}{|c|}{ Uso de condón } \\
\hline & & & & & & & en la pr & a relación** \\
\hline & & & $\mathrm{n}$ total & & & & \multirow{3}{*}{$\begin{array}{l}\text { sexualmente } \\
\text { activos }\end{array}$} & \\
\hline & \multicolumn{2}{|c|}{ Actividad sexual* } & sexualmente & \% sí usó & \multicolumn{2}{|c|}{ Actividad sexual* } & & \multirow{2}{*}{$\begin{array}{l}\text { \% sí usó } \\
\text { condón }\end{array}$} \\
\hline & $\mathrm{n}$ total & $\%$ activos & activos & condón & $\mathrm{n}$ total & $\%$ activos & & \\
\hline I Noroeste & 814 & 18.7 & 147 & 50.6 & 770 & 8.3 & 64 & 18.6 \\
\hline II Norte & 830 & 13.4 & 109 & 36.0 & 803 & 6.4 & 50 & 11.9 \\
\hline III N oreste & 463 & 14.5 & 67 & 52.8 & 438 & 8.9 & 37 & 38.2 \\
\hline IV Centro D & 1318 & 12.9 & 169 & 68.1 & 1360 & 7.0 & 96 & 16.4 \\
\hline V Centro E & 2512 & 14.7 & 369 & 49.5 & 2476 & 8.2 & 203 & 27.1 \\
\hline VI Sur & 745 & 13.8 & 102 & 51.2 & 799 & 4.4 & 34 & 24.3 \\
\hline VII Este & 480 & 12.3 & 59 & 47.0 & 513 & 2.9 & 15 & 25.0 \\
\hline VIII Península & 252 & 12.7 & 32 & 33.1 & 223 & 3.6 & 9 & 18.3 \\
\hline Total del país & 7414 & 14.3 & 1054 & 51.0 & 7382 & 6.9 & 508 & 23.0 \\
\hline \multicolumn{9}{|c|}{$\begin{array}{l}\text { Conformación de las Regiones } 17 \\
\text { Región I (N oroeste): Baja California, Baja California Sur, N ayarit, Sinaloa, Sonora } \\
\text { Región II (N orte): C oahuila, Chihuahua, Durango, San Luis Potosí, Zacatecas } \\
\text { Región III (N oreste): N uevo León,Tamaulipas } \\
\text { Región IV (Centro-D): A guascalientes, Colima, Guanajuato, Jalisco, Michoacán } \\
\text { Región V (C entro-E): D istrito Federal, H idalgo, Estado de México, Morelos, Puebla, Q uerétaro,T Taxcala } \\
\text { Región VI (Sur): C hiapas, Guerrero, O axaca } \\
\text { Región VII (Este):Tabasco,Veracruz } \\
\text { Región VIII (Península): C ampeche, Q uintana Roo,Yucatán } \\
\text { N ota: Ios casos sin respuesta no están incluidos en el cuadro }\end{array}$} \\
\hline
\end{tabular}

Fuente: cálculos propios a partir de SSA, EN SA 2000 
que menor actividad sexual presentan de hombres y de mujeres son Región VII Este y Región VIII Península.

El uso del condón en la primera relación sexual entre adolescentes solteros muestra grandes diferencias entre los dos sexos. Mientras que uno de cada dos hombres sexualmente activos dijo haberlo usado en la primera relación sexual (50.9\%), sólo una de cada cinco mujeres reportó su uso $(22.9 \%)(p<0.00)$. Diferenciando el ámbito rural y el urbano, los hombres rurales tienden a usar menos que los urbanos (41.3\% vs. 55.1\%, $p<0.00$ ), pero la mayor diferencia se advierte en las mujeres, donde sólo una de cada diez dijo haber usado condón en su debut sexual (11.6\% vs. 27.9\%, $p<0.00)$. En el nivel regional, una mayor proporción de uso de condón no está asociada con un mayor porcentaje de sexualmente activos (cuadro II) (para los hombres $p<0.14$; para las mujeres $p<0.27$ ). En los hombres de la Región IV Centro D la prevalencia de uso del condón fue de $68.1 \%$, y la mayor prevalencia en mujeres se observa en la Región III Noreste, con un nivel de $38.2 \%$.

Una variable interesante que permite relacionar el comportamiento de prevención pasado con el presente es el uso de método actual, pero desafortunadamente tuvo un bajo porcentaje de respuestas $(14.5 \%$ respondió la pregunta). Sin embargo, a pesar del bajo número de respuestas, las diferencias entre hombres y mujeres de este pequeño grupo fueron estadísticamente significativas. Se advierte que entre aquellos adolescentes solteros que se protegieron con condón en la primera relación sexual, son las mujeres las que siguen utilizándolo más como método en la actualidad (69\% de los hombres y $94 \%$ de las mujeres dijeron utilizarlo como método actual; $p<0.01$ ), en tanto que $31 \%$ de los hombres ha cambiado al uso de otros métodos que sólo protegen contra embarazos (pastillas e inyecciones como primeras opciones).

Acerca de quién decidió el uso del condón en esa primera relación sexual (figura I), es claro que tanto hombres como mujeres solteros están de acuerdo en que, en general, ella no toma la iniciativa, aunque las mujeres consideran tener más participación al respecto $(5.9 \%$ de los hombres vs $9.5 \%$ de las mujeres). También están de acuerdo en que en un alto porcentaje lo decide el hombre (57.2\% de los hombres y $41.4 \%$ de las mujeres). Pero hay diferencias sobre si consideran que la decisión fue conjunta o lo decidió él. Las mujeres tienden a afirmar que la decisión es más compartida (49.1\%), en tanto que los hombres sólo creen que fue compartida en 36.9\%. Estas diferencias fueron estadísticamente significativas $(p<0.01)$.

Respecto del conocimiento que los adolescentes tienen sobre métodos de protección contra las ITS resalta
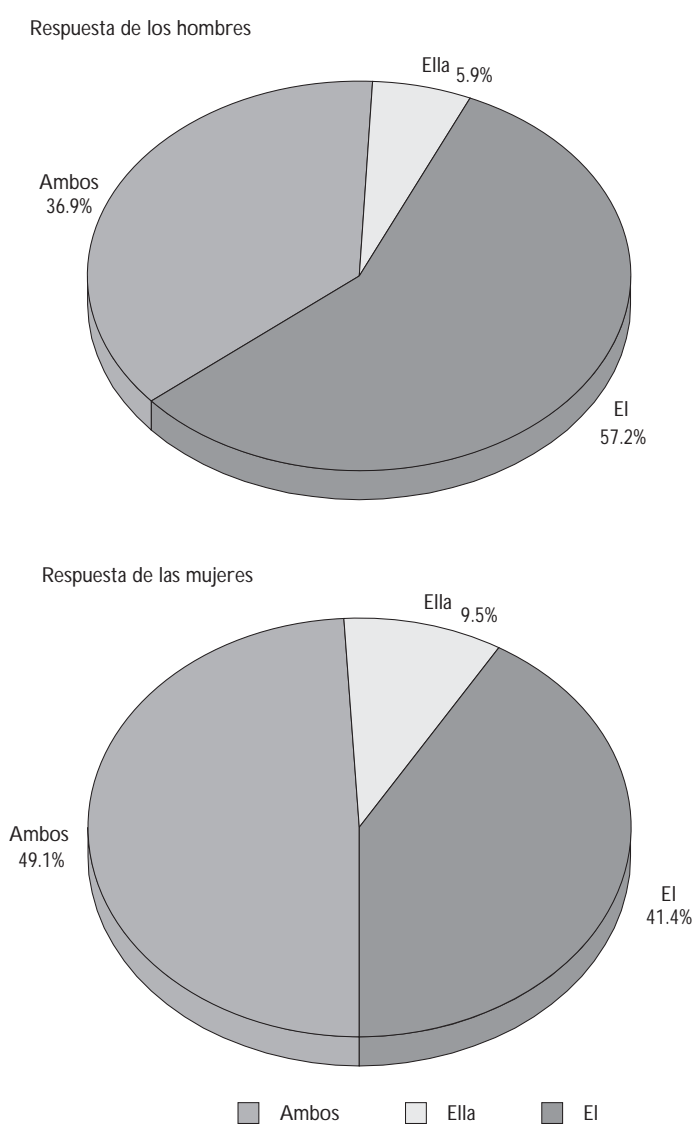

$(p<0.01)$

Fuente: cálculos propios a partir de Secretaría de Salud, México, EN SA 2000

Figura 1. Quién decidió el uso de condón en la PRIMERA RELACIÓN SEXUAL.AdOLESCENTES 12 A 19 AÑos de EDAD, SOLTEROS

que entre los solteros sexualmente activos casi uno de cada cuatro hombres $(23.8 \%)$ y casi una de cada dos mujeres $(40.9 \%)$ no conoce ningún método $(p<0.00)$. De aquellos que mencionaron conocer métodos de prevención de ITS, la gran mayoría consideró el condón como mejor método ( $88 \%$ de los hombres y $92 \%$ de las mujeres). El peso de la abstención fue mayor en las respuestas de los hombres que de las mujeres ( $9 \%$ versus $6 \%$ ). Finalmente, la monogamia (catalogada en la encuesta como relación fiel), es considerada como el mejor método de prevención de ITS en una proporción mucho menor que las dos categorías anteriores, pero mayor en hombres que en mujeres (2.5\% contra $1.7 \%$ ). Las diferencias entre hombres y mujeres respecto al mejor método no fueron estadísticamente significativas $(p<0.16)$.

La ENSA 2000 preguntó a los adolescentes de 12 a 19 años de edad si habían tenido alguna enfermedad de 
transmisión sexual en los últimos 12 meses. Se acepta que el reporte de los entrevistados sobre este tipo de enfermedades subestima el número de casos reales, comparado con los estudios de prevalencias basados en pruebas de laboratorio, sobre todo teniendo en cuenta que muchas de estas infecciones pueden ser asintomáticas. A pesar de esta subestimación, y cuidando de no considerar los niveles como marcadores de prevalencias, esta información proporciona un indicador grueso de las diferencias entre hombres y mujeres. De los hombres solteros sexualmente activos, $1.6 \%$ reportó haber tenido alguna enfermedad de transmisión sexual en el último año. En contraste, las mujeres reportaron la mitad $(0.8 \%)$ $(p<0.06)$ (cuadro III).

Presentamos a continuación los resultados del análisis exploratorio de los factores asociados con el uso o no uso de condón en la primera relación sexual para el total de la población adolescente de 12 a 19 años. Para ello se utilizó la técnica de regresión logística multivariada donde la variable dependiente fue el uso o no del condón en la primera relación sexual entre los adolescentes mexicanos. Múltiples son los factores que intervienen en la prevención de las ITS a través del uso del condón, sin embargo, en el presente análisis nos enfocaremos a cuatro tipos de factores: demográficos, socioeconómicos, culturales y cognitivos.

Las variables demográficas consideradas son el sexo, la edad de la primera relación sexual, y el estado civil. Se ha observado que las variables demográficas, por ejemplo, las características personales del individuo, principalmente la edad y otras asociadas con la exposición al tiempo de ocurrencia de un suceso o acción, ${ }^{18}$ tienen una gran influencia sobre un suceso en cuestión,

\section{Cuadro III \\ Reporte de enfermedad de transmisión SEXUAL en el Último año. Adolescentes 12-19 años DE EDAD SEXUALMENTE ACTIVOS}

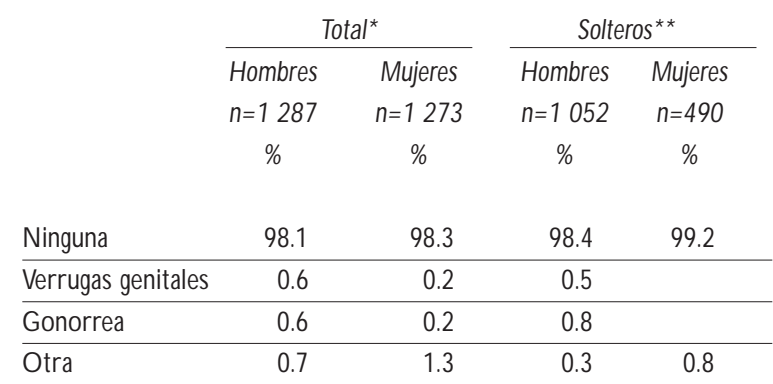

Fuente: cálculos propios a partir de SSA, EN SA 2000.

$*(p<0.02)$

$* *(p<0.06)$ en este caso, el uso del condón. Sin embargo, la variable sobre estado civil fue incluida pero sólo como un control demográfico, por no tener la información acerca del estado civil al momento de la primera relación sexual sino al momento de la encuesta.

Las variables socioeconómicas consideradas en el análisis multivariado son escolaridad y lugar de residencia (urbano/rural) del adolescente. En los estudios demográficos y epidemiológicos, el nivel de escolaridad siempre ha mostrado tener una influencia en el comportamiento individual, ${ }^{19} \mathrm{y}$ es de esperarse la misma influencia al examinar las prácticas sexuales de prevención. El resultado esperado es que los grupos de baja educación y bajos recursos, tengan una menor prevención de los riesgos sexuales comparados con la población en mejores condiciones socioeconómicas. ${ }^{20}$ De igual forma, el contexto urbano o rural será determinante en el comportamiento de los jóvenes, por ser tan contrastantes las opciones de servicios, conocimientos, valores y actitudes en ambos ámbitos.

Aunque son múltiples los factores culturales que podrían considerarse relacionados con el uso del condón, dada la limitación de la encuesta sólo hemos incluido la variable "hablar lengua indígena". Dicha variable se usa aquí como una aproximación a los grupos étnicos que por su cultura tan diferente y aislamiento de los beneficios que provee la sociedad moderna pudieran estar en desventaja respecto de lograr una actividad sexual protegida. ${ }^{21}$

Finalmente, los factores cognitivos también fueron incluidos en el análisis. El conocimiento de cómo prevenir las ITS es un factor antecedente. Esto significa que el conocimiento de que las relaciones sexuales pueden acarrear consigo riesgos de infección y de que el uso del condón previene la transmisión de esas infecciones son pre-requisitos para poder tener sexo protegido. Sin embargo, aun teniendo los conocimientos, ésta no es una condición suficiente para que el sexo protegido ocurra. De todas maneras, suponemos que el contar con información de métodos de prevención de ITS hace más probable el uso del condón.

El cuadro IV muestra los resultados del análisis multivariado. Con respecto a los factores demográficos el sexo es un factor determinante. Los hombres usan 3.5 veces más el condón en la primera relación sexual que las mujeres. La edad de la primera relación es estadísticamente significativa en sentido positivo. Una vez controlados los otros factores, se observa que el retraso en la edad de debut sexual está asociado con un mayor uso del condón. El efecto es fuerte pues se observa que es 3.5 veces más probable que los jóvenes de 18 a 19 años de edad usen condón en la primera relación sexual que los jóvenes de 12 a 13 años. 


\section{Cuadro IV \\ Características asociadas con el uso de condón en La primera relación SEXUAL.Adolescentes SEXUALMENTE ACTIVOS 12 A 19 AÑOS DE EDAD}

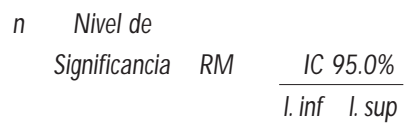

\begin{tabular}{|c|c|c|c|c|}
\hline \multicolumn{5}{|l|}{ Sexo } \\
\hline Mujer & \multicolumn{2}{|l|}{1453} & \multicolumn{2}{|l|}{1.0} \\
\hline Hombre & 1099 & 0.000 & 3.5 & $2.8 \quad 4.3$ \\
\hline \multicolumn{5}{|c|}{ Edad (en la primera relación } \\
\hline \multicolumn{5}{|c|}{ sexual)* } \\
\hline $12-13$ & 189 & 0.000 & 1.0 & \\
\hline $14-15$ & 828 & 0.001 & 2.1 & $1.4 \quad 3.3$ \\
\hline $16-17$ & 1095 & 0.000 & 2.7 & $\begin{array}{ll}1.7 \quad 4.1 \\
\end{array}$ \\
\hline $18-19$ & 379 & 0.000 & 3.5 & 2.25 .7 \\
\hline \multicolumn{5}{|l|}{ Estado civil } \\
\hline No soltero & 1159 & & 1.0 & \\
\hline Soltero & 1393 & 0.000 & 2.6 & 23.2 \\
\hline \multicolumn{5}{|l|}{ Escolaridad } \\
\hline Hasta primaria & 790 & 0.000 & 1.0 & \\
\hline Secundaria & 1038 & 0.000 & 1.8 & $1.3 \quad 2.3$ \\
\hline Preparatoria y más & 675 & 0.000 & 2.6 & $2.0 \quad 3.5$ \\
\hline \multicolumn{5}{|l|}{ Residencia } \\
\hline Rural & 1078 & & 1.0 & \\
\hline Urbana & 1474 & 0.000 & 1.8 & $1.4 \quad 2.2$ \\
\hline \multicolumn{5}{|l|}{ Lengua indígena } \\
\hline Sí habla & 141 & & 1.0 & \\
\hline No habla & 2411 & 0.000 & 5.3 & 2.113 .3 \\
\hline \multicolumn{5}{|c|}{ Conoce métodos prevención de ITS } \\
\hline Sí conoce & 1641 & & 1.0 & \\
\hline No conoce & 898 & 0.369 & 0.9 & $0.7 \quad 1.1$ \\
\hline
\end{tabular}

Los factores estructurales, como la educación y el contexto rural/urbano, muestran estar influyendo significativamente el sexo protegido en la primera relación sexual. Tener un nivel escolar de secundaria incrementa casi dos veces la probabilidad de uso del condón en el debut sexual, y 2.6 veces para aquellos jóvenes con preparatoria o mayor escolaridad, comparados con quienes sólo alcanzaron un nivel de escolaridad hasta primaria. En cuanto a la influencia del contexto rural o urbano, los jóvenes que residen en áreas urbanas tienen una probabilidad mayor en 1.8 veces de usar condón en la primera relación sexual que quienes residen en el área rural.

La variable hablar lengua indígena es la que muestra las mayores razones de momios en el modelo. La población que no habla lengua indígena tiene cinco veces más probabilidad de usar condón en la primera relación sexual que los adolescentes que hablan lengua indígena, una vez controlados los otros factores.

La variable que se refiere al conocimiento de métodos de prevención de enfermedades de transmisión sexual no resultó estadísticamente significativa.

\section{Discusión}

El porcentaje de adolescentes sexualmente activos entre los 15 y los 19 años de edad varía en distintas regiones del mundo. América Latina se caracteriza por un inicio sexual en edades más tempranas que otras regiones en desarrollo como Asia, pero mucho más tardío que Africa. ${ }^{22}$ Dentro de América Latina los datos de la ENSA 2000 aquí analizados para México muestran que este país se encuentra entre los de menor proporción de sexualmente activos, sobre todo por el comportamiento de las mujeres. ${ }^{22}$

En cuanto al uso del condón la tendencia es errática dentro de las distintas regiones en desarrollo. Al parecer el patrón indica que es América Latina la región con mayor porcentaje de uso en la primera relación sexual. Dentro de América Latina, México tiene un nivel más bajo de uso comparado con otros países como Argentina. ${ }^{23}$

Con la información de los últimos dos decenios es difícil precisar el porcentaje de adolescentes sexualmente activos y de protección debido a la dispersión entre los resultados a los cuales llegan las distintas fuentes de datos. Sin embargo, se puede apreciar una tendencia general en las estadísticas del pasado de un porcentaje de actividad sexual mayor en los hombres que en las mujeres. Los resultados de la ENSA 2000 que aquí presentamos muestran que el diferencial de actividad sexual por sexo se ha reducido. Dentro del país encontramos diferencias regionales respecto de la proporción de actividad sexual entre los adolescentes solteros; no obstante, el diferencial por sexo se mantiene en todas las regiones.

Respecto a la edad media para la primera relación sexual, los datos obtenidos en la ENSA 2000 siguen el patrón de las encuestas previas: las mujeres inician su vida sexual un poco más tarde que los hombres, pero, aparentemente, estarían iniciando, tanto hombres como mujeres, en una edad más temprana que la estimada con anterioridad. Una vez más hay que destacar las 
diferencias en las edades consideradas por cada encuesta, que dificultan la comparación.

Desde las primeras estimaciones del uso del condón por los jóvenes solteros realizadas a mediados de la década de 1980, la curva de uso del condón ha ido incrementándose, pasando de $7 \%$ en $1985^{8}$ a $51 \%$ en el año 2000 (ENSA 2000) entre los hombres, y de 5 a 23\% entre las mujeres (cuadro I). Una posible explicación para este incremento es el efecto de las campañas de prevención que se desarrollaron a partir de la identificación del SIDA como problema de salud pública, especialmente después de la década de 1990, y el cambio en la curricula escolar a partir de 1998 en la cual se incluye de manera obligatoria la educación sexual en las primarias y secundarias de todo el país. Los bajos valores de la encuesta de 1985 corresponden a un periodo anterior a la preocupación intensiva por la pandemia del SIDA en México.

Otra tendencia observada en las estimaciones de uso del condón es que permanece el diferencial por sexo a través del tiempo. Las mujeres reportan en la ENSA 2000 menos uso que los hombres, al igual que en las otras encuestas analizadas (cuadro I), incluso si se controlan otros factores (cuadro IV). Muchas de estas diferencias podrían derivarse de un sistema sexogénero $^{24}$ que influye, tanto en las actitudes como en las conductas sexuales y de prevención de ITS. En una sociedad como México, donde la brecha de género es grande y la mujer suele tener un papel pasivo en cuanto a las circunstancias de la relación sexual, la hipótesis es que el uso del condón es facultad de los hombres.

Para poder entender quién utiliza el condón y quién no existe una multiplicidad de factores, algunos más inmediatos y que anteceden al acto sexual (como las características individuales), y otros que tienen un carácter relacional y suponen, tanto conocer las características de la pareja sexual como las circunstancias en las que ocurre la relación sexual. ${ }^{1}$ Con la información disponible en la ENSA 2000, hemos considerado sólo cuatro tipos de factores relevantes referidos a las características del individuo. La ENSA 2000 no aporta información sobre la pareja sexual o las circunstancias en que ocurrió la interacción sexual. A pesar de estas limitaciones, los cuatro tipos de factores estudiados muestran que tienen una influencia en la práctica preventiva respecto a las ITS.

Nuestro análisis muestra que iniciar la vida sexual a una mayor edad incrementa el uso del condón de manera sustancial. Esto significa que quienes la inician muy jóvenes tienen menor probabilidad de usarlo en su primera relación, y, por tanto, un riesgo mayor de contraer una ITS. Este hallazgo apunta a la necesidad de desarrollar políticas que promuevan el uso del condón en edades aún antes del inicio de las relaciones sexuales. Asimismo, un mayor nivel de escolaridad en nuestro modelo se asocia con un mayor uso del condón. La literatura precedente señala la importancia de la educación como un factor que incrementa la percepción del riesgo de las ITS. ${ }^{25}$

También en México se encuentra un diferencial en el uso del condón en la primera relación sexual entre los adolescentes de las áreas urbanas respecto de los de áreas rurales, aun cuando se controle por otras variables como escolaridad y edad. El menor uso en áreas rurales puede deberse a las dificultades de distribución y de acceso a los condones en localidades alejadas de los grandes centros, sin excluir factores culturales como una menor percepción del riesgo (queda por demostrar si existe un menor riesgo de contraer ITS para los adolescentes rurales).

Otro hallazgo de importancia es que el conocimiento de métodos de prevención de ITS no influyó en la decisión de haber usado el condón en la primera relación sexual. Esto nos lleva a pensar en dos razones que pudieran explicar la falta de diferencial. Por un lado, se puede considerar que la información que tienen los jóvenes sobre los métodos para prevenir las ITS es inadecuada. Otras razones que pueden estar afectando es que además del conocimiento son necesarias otras habilidades como la capacidad de negociar, la percepción positiva del condón, la accesibilidad, etcétera. ${ }^{26}$ Otras investigaciones han dirigido su atención a esta brecha entre conocimiento y práctica, sugiriendo que existen algunos obstáculos culturales que impiden aplicar los saberes recibidos. ${ }^{27-29}$ Nuevos estudios podrán ayudar a diseñar programas de prevención que cierren esta distancia.

El perfil del adolescente que usó condón en la primera relación y que está en condiciones de prevenir una ITS, de acuerdo con este análisis de la ENSA 2000, es ser adolescente que inicia la vida sexual a una mayor edad, de residencia urbana, que no habla lengua indígena, mediana o altamente escolarizado, o de sexo masculino. Conocer el perfil del adolescente que usó condón en su debut sexual evidencia, a su vez, al tipo de adolescentes que tuvo menos probabilidad de usarlo: adolescentes que inician su vida sexual más temprano, que hablan lengua indígena, que viven en áreas rurales, menos escolarizados y las mujeres. Este hallazgo alerta sobre la necesidad de diseñar políticas específicas de prevención de ITS que se dirijan a esta población que inicia su vida sexual en condiciones de mayor riesgo. 


\section{Referencias}

1. Juárez F. Salud Reproductiva de los jóvenes: teorías y evidencia. Rabell C, Z avala-de Cosío E, ed. La fecundidad en condiciones de pobreza: una visión internacional. Instituto de Investigaciones Sociales, UN AM, México, 2002.

2. Gardner R, Blackburn RD, U padhyay UD. Condones: cómo cerrar la brecha entre el uso y la necesidad. Population Reports, Serie H, N 0. 9, Baltimore, John Hopkins U niversity School of Public Health, Population Information Program, abril de 1999.

3. Del Río-Chiriboga C, U ribe-Zúñiga P. Prevención de enfermedades de transmisión sexual y SIDA mediante el uso del condón. Salud Publica Mex 1993; 35:508-517.

4. Cates W. The N IH Condom Report:The Glass is $90 \%$ Full. Family Planning Perspectives 2001;33(5): 231-233.

5.Arredondo-García JL, N arcio-Reyes ML, C asanova-Román G, Figueroa-D amián R. Enfermedades transmitidas sexualmente (ETS) en un grupo de adolescentes que asisten a un Instituto de Atención Perinatal. Gac Med Mex 1993;129(1):75-79.

6.Valdespino-Gómez JL, G arcía-García ML, D el Río-C hiriboga C, C ruzPalacios C, Loo-Méndez E, López-Sotelo A. Las enfermedades de transmisión sexual y la epidemia de VIH/SIDA. Salud Publica Mex 1995; 37:549-555.

7. Conde-González C, U ribe-Salas F. G onorrea: la perspectiva clásica y la actual. Salud Publica Mex 1997; 39:573-579.

8. Morris L. D etermining male fertility Through Surveys:Young adult reproductive health surveys in Latin American. Ponencia presentada en la General Conference of the International Union for the Scientific Study of Population, 1993.

9. Consejo $\mathrm{N}$ acional de Población. Encuesta $\mathrm{N}$ acional sobre Sexualidad y Familia en Jóvenes de Educación Media Superior. México, DF: Conapo, 1988.

10. Secretaría de Salud. Informe de la Encuesta sobre el

Comportamiento Reproductivo de los Adolescentes y Jóvenes del A rea Metropolitana de la Ciudad de México. México, DF: SSA, 1988.

11. Consejo N acional para la Prevención y el Control del VIH/SIDA. Encuesta sobre Comportamiento Sexual en la Ciudad de México. México, DF: CO N ASIDA, 1994.

12. Fundación Mexicana para la Planeación Familiar, AC. Encuesta para el programa Gente Joven 1999. México, DF:MEX FAM, 1999.

13. Instituto Mexicano de la Juventud. Encuesta $\mathrm{N}$ acional de Juventud 2000. Resultados Básicos. México, D F:IMJ/SEP, 2002.

14. Sánchez-Alemán M. Encuesta de Jóvenes Universitarios de la UA EM

2001. En: Prevalencia y Factores asociados a la Infección por Treponema Pallidum, virus del herpes simple tipo 2 y virus del papiloma humano en estudiantes universitarios. Tesis de Maestría en Ciencias de la Salud. Instituto N acional de Salud Pública, Cuernavaca, 2001.

15. Huerta R, Malacara J. Prácticas sexuales en adolescentes de áreas marginadas: su asociación con la estructura y función familiar. Carta de Población: Boletín 0 ficial del Grupo Académico de A poyo a Programas de Población, Año 2, no9, septiembre de 1995:1-7.

16.Valdespino JL, Plaiz G, López-Barajas MP, Mendoza L, Palma 0 , Velázquez 0 et al. Encuesta $\mathrm{N}$ acional de Salud 2000.Vivienda, población y utilización de servicios de salud. Cuernavaca, Morelos, México, Instituto N acional de Salud Pública, 2003.

17. Rivera S. Desarrollo y urbanización regional en México, 1970-1990. Estudios D emográficos y U rbanos 27, 1994;9(3):657-679.

18. Pressat R. El análisis demográfico. Métodos, resultados, aplicaciones. México DF: Fondo de Cultura Económica, 2000.

19. C astro T, Juárez F. W omen's education and fertility in Latin A merica. Int Fam Plann Perspectives 1995;21(2):52-57,80.

20. Singh S, Darroch J, Frost J. Socioeconomic disadvantage and adolescent women's sexual and reproductive behaviour:The $C$ ase of Five D eveloped Countries. Family Planning Perspectives 2001;33(6): 258-259.

21.Valdés LM. El perfil demográfico de los indios mexicanos. México DF: Siglo Veintiuno, 1989.

22. Brown AD, Jejeebhoy S, Shah I,Yount K. Sexual relations among young people in developing countries: Evidence from W HO case studies. W orld Health O rganization, W HO /RHR/01.8, 2001.

23. UN AID S/W HO . Argentina Epidemiological Fact Sheets on HIV/AIDS and Sexually Transmitted Infections. 2002 U pdate.

24. Rubín G. El tráfico de mujeres: notas sobre la 'economía política' del sexo. En Lamas M, comp. El género. La construcción cultural de la diferencia sexual. 2ª Ed. México DF, PUEG -Porrúa, 2000:35-96. 25. United N ations, HIV/AIDS Awareness and Behaviour, Department Economic and Social Affairs, Population D ivision, N ueva York, 2002. 26. Moatti JP, Beltzer N, Dab W. Les modèles d'analyse des comportements à risque face à l'infection àVIH: Une conception trop étroite de la rationalité. Population 1993;5:1505-1534.

27. Castro-Vázquez G. Masculinity and condom use among Mexican teenagers:The Escuela N acional Preparatoria N 0 1's case. Gender and Education, 2000;12(4):479-492.

28. $G$ ayet $C$, Juárez $F$.The influence of culture as an obstacle to condom use among young Mexicans, Collection of Papers from the XIV International AID S Conference, Barcelona, Spain, 7-12 July 2002. Monduzzi Editore, International Proceedings Division, Italia, 2002. 29. Pedrosa L,Vallejo M. Entorno social, comportamiento sexual y reproductivo en la primera relación sexual de adolescentes estudiantes de escuelas públicas y privadas. En Stern C, Echarri C, comps. Salud Reproductiva y Sociedad. Resultados de investigación. México D.F.: El Colegio de México, 2000. 